\title{
Effect of varying poisson ratio on thermally induced vibrations of non-homogeneous rectangular plate
}

\author{
Anupam Khanna ${ }^{1 *}$, Narinder Kaur ${ }^{2}$, Ashish Kumar Sharma ${ }^{3}$ \\ ${ }^{1}$ *Department of Mathematics, Maharishi Markandeshwar University, Mullana, Ambala, Haryana, India \\ ${ }^{2}$ Department of Mathematics, Maharishi Markandeshwar University, Mullana, Ambala, Haryana, India \\ ${ }^{3}$ Department of Mathematics, ManavBharti University, Solan (H.P.), India \\ 1*anupam_rajie@yahoo.co.in, 2narinder89.kaur@gmail.com, ${ }^{3}$ ashishk482@gmail.com
}

\begin{abstract}
A mathematical model will help the engineers in science and technology applications. In this paper, authors studied a temperature-thickness coupling problem of a non-homogeneous rectangular plate in which temperature varies bilinearly \& thickness varies linearly in x-direction. Due to non-homogeneity, it is considered that poisson ratio varies exponentially in $\mathrm{x}$ direction. Ray-Leigh Ritz method has been adopted to calculate the time period for fixed two modes of vibration for different values of aspect ratio, thermal gradient and taper constants. All results are shown in graphs.
\end{abstract}

Keywords: Vibration, Thermal gradient, Taper constant, Aspect Ratio, Time Period

\section{Introduction}

There has been amazing discoveries in the field of vibration research, drawing the attention of scientists and design engineers to study tremendous effect of vibrational behaviour in engineering and modern technology. In the field of mechanical engineering, new discoveries can't be possible without considering the effect of vibration as almost all machines and engineering structures experience. Study of vibration is not just confined to science but also our day to day life. From its constructive aspects in aircrafts engineering, space technology, etc., to the destructive aspects e.g. earthquake, satellite; nothing is untouched by vibration effects.

Plates of variable thickness are commonly used in many engineering applications like spacecrafts, submarine, nuclear reactor ships etc. So it is the need of the hour to get deeper knowledge of the plate's behaviour and their characteristics, which would in turn help to perceive their potential in many fields.

In the modern technology, an interest towards the effect of high temperatures on non-homogeneous rectangular plates of variable thickness is developed due to applications in various engineering branches such as nuclear, power plants, aeronautical, chemical etc. where metals and their alloys exhibits visco-elastic behaviour. The reason for these is that during heating up periods, structures are exposed to high intensity heat fluxes and material properties undergo significant changes; in particular thermal effect cannot be negligible. A lot of literature is available in one dimensional variation in temperature with thickness variation of plates, but negligible work is found in two dimensional temperature variations.

Cheung and Zhou (1999) have studied several aspects concerning the formulation and the solution of amplitude equations for free vibration of systems with cubic non-linearity. Dhotarad and Ganesan (1978) analysed the dynamic free response of thin rectangular plates subjected to one and two dimensional steady state temperature distributions. Gupta et al. (2007) had

Research article

CCIndian Society for Education and Environment (iSee) analysed the effect of non-homogeneity on thermally induced vibration of orthotropic visco-elastic rectangular plate of linearly varying thickness. Gupta et al. (2009) had evaluated time period and deflection for the first two modes of vibration of visco-elastic rectangular plate and for various bi-linearly thickness variation. Jain and Soni (1973) had studied the free vibrations of rectangular plates with thickness varying parabolically. Kumar and Sanjay (2003) had analysed the vibration of visco-elastic isotropic rectangular plate with varying thickness in two directions i.e. linearly in one and parabolically in other direction. Khanna and Anupam (2005) had studied the vibrations on visco-elastic rectangular plate with the variable thickness without considering thermal effects. Lal Roshan and Dhanpati (2009) had analysed the vibrations of non-homogeneous orthotropic rectangular plates of varying thickness with two opposite simply supported edges and resting on two-parameter foundation. Laura et al. (1979) had studied the transverse vibrations of rectangular plates with linear variation of the thickness in the $x$ and $y$ directions. Leissa (1987) had helped the many researchers by collecting the various research papers which show the latest researches done in the field of vibration of plates without considered two dimensional thermal effects. Leissa (1987) had evaluated the effect of thermal gradient on the vibration of parallelogram plate with linearly varying thickness in both direction and thermal effect in linear form only. Singh and Saxena V (1996) had studied the transverse vibrations of a rectangular plate of variable thickness with different combinations of boundary conditions at the four edges. Tomar and Gupta (1983) had evaluated the thermal gradient effect on the vibration of a rectangular plate having bi-directional variation thickness. Tomar and Gupta (1985) had studied the effect of thermal gradient on frequencies of an orthotropic rectangular plate whose thickness varies in two directions. Li (2005) gave an analysis on modal characteristics on vibrations of rectangular plate with general elastic supports along its edges. 
In this research paper, our aim is to provide a theoretical and authentic mathematical model for analysing the vibrational behaviour of non-homogeneous rectangular plate of varying thickness with two dimensional temperature variations. Temperature variation is considered bi-linearly i.e. linearly in $x \& y$ direction \& thickness variation is assumed linearly in $x$ direction. The rectangular plate is clamped at all four edges. Due to Non homogeneity in the material of plate, it is considered that poisson ratio varies exponentially along $x$ direction. Ray-Leigh Ritz method has been used to calculate both modes of time period for different values of aspect ratio, taper constant, thermal gradient and nonhomogeneity constant.

\section{Equation of motion and analysis}

Differential equation of motion for rectangular plate of variable thickness for free vibration of variable thickness is given by (1):

$$
\begin{aligned}
& {\left[D_{1}\left(W_{x x x x}+2 W_{x x y y}+W_{y y y y}\right)+2 D_{1 x}\left(W_{x x x}+W_{x y y}\right)+\right.} \\
& 2 D_{1 y}\left(W_{y y y}+W_{y x x}\right)+D_{1 x x}\left(W_{x x}+v W_{y y}\right)+ \\
& \left.D_{1 y y}\left(W_{y y}+W_{x x x}\right)+2(1-v) D_{1, x y} W_{x y}\right]-\rho p^{2} h W=0
\end{aligned}
$$

which is a fourth-order differential equation of transverse motion for non-homogenous plate of variable thickness. Here $D_{1}$ is flexural rigidity of rectangular plate i.e.

$D_{1}=E g^{3} / 12\left(1-v^{2}\right)$

and corresponding two-term deflection function is taken as follows:

$W=$

$[(x / a)(y / b)(1-x / a)(1-y / b)]^{2}\left[A_{1}+A_{2}(x / a)(y / b)(1-\right.$ $x / a)(1-y / b)$

Assuming that the rectangular plate of engineering material has a steady two dimensional linear temperature distribution i.e.

$\tau=\tau_{0}(1-x / a)(1-y / b)$

where $\tau$ denotes the temperature excess above the reference temperature at any point on the plate and $\tau_{0}$ denotes the temperature at any point on the boundary of plate and " $a$ " is the length of a side of rectangular plate and " $b$ " denote the width of rectangular plate.

The temperature dependence of the modulus of elasticity for most of engineering materials can be expressed in this form,

$E=E_{0}(1-\gamma \tau)$

where $E_{0}$ is the value of the Young's modulus at reference temperature i.e. $\tau=0$ and $\gamma$ is the slope of the variation of $E$ with $\tau$. The modulus variation (5) become

$E=E_{0}(1-\alpha(1-x / a)(1-y / b))$

where $\alpha=\gamma \tau_{0}(0 \leq \alpha<1)$ is thermal gradient. It is assumed that thickness varies linearly in one direction as shown below: $g=g_{0}(1+\beta x / a)$

where, $\beta$ is taper constant in $\mathrm{x}$-direction and $\mathrm{g}=\mathrm{g}_{0}$ at $\mathrm{x}=0$.

Also, It is assumed that Poisson ratio of material varies exponentially in $\mathrm{x}$-direction as shown below:

Research article

(C)Indian Society for Education and Environment (iSee)

"Thermal gradient"

http://www.indjst.org
Vol. 5 No. 9 (Sep. 2012) ISSN: 0974- 6846 $v=v_{0} e^{\alpha_{1} x / \alpha}$

In order to avoid infinite terms in the expansion of $e^{\alpha_{1} x / \alpha}$, authors consider first six terms of the expansion s.t. $v=v_{0} e^{\alpha_{1} x / a}=v_{0}\left[1+\left(\alpha_{1} x / a\right) / 1 !+\left(\alpha_{1} x / a\right)^{2} / 2 !+\left(\alpha_{1} x / a\right)^{3} / 3 !\right.$ $\left.+\left(a_{1} \times / a\right)^{4} / 4 !+\left(a_{1} x / a\right)^{5} / 5 !\right](8)$

Put the value of $E \& g$ from equation $(6),(7) \&(8)$ in the equation (2), one obtain

$$
\begin{aligned}
& D_{1}=\left(E_{0}(1-\alpha(1-x / a)(1-y / b))\right)\left(g_{0}(1+\beta x / a)\right)^{3} / \\
& 12\left(1-v_{0}^{2} e^{\frac{2 a_{1} x}{a}}\right)
\end{aligned}
$$

Rayleigh-Aitz technique is applied to solve the frequency equation. In this method, one requires maximum strain energy must be equal to the maximum kinetic energy. So it is necessary for the problem under consideration that $\delta\left(P^{*}-K^{*}\right)=0$

for arbitrary variations of $W$ satisfying relevant geometrical boundary conditions.

Since the plate is assumed as clamped at all the four edges, so the boundary conditions are

$\mathrm{W}=W_{x}=0$ at $x=0, a, W=W_{y}=0$ at $y=0, \mathrm{~b}(11)$

Now assuming the non-dimensional variabies as $X=x / a, Y=y / a$

The kinetic energy $\mathrm{K}^{\star}$ and strain energy $\mathrm{P}^{\star}$ are

$K^{*}=1 / 2 \rho p^{2} a^{2} g_{0} \int_{0}^{1} \int_{0}^{b / a}(1+\beta X) W^{2} d Y d X$

$P^{*}=Q \int_{0}^{1} \int_{0}^{b / a}\left\{(1-\alpha(1-X)(1-a / b Y))(1+\beta X)^{3} /(1-\right.$

$\nu 02 e a 1 X)\} W, X X 2+W, Y Y 2+2 v 0 e a 1 X W, X X W, Y Y+2(1-\nu 0 e$ $a(X)(W, X Y) Z) d X d Y$

where, $Q=E_{0} g_{0}{ }^{3} / 24 a^{2}$

Now put the value of $K^{*}$ and $P^{*}$ from eq (13) and (14) in eq (10).After putting all the values we get

$\left(P^{*}-\lambda^{2} K^{*}\right)=0$

Here $\lambda^{2}=12 \rho p^{2} a^{4} / E_{0} g_{0}^{2}$ is a frequency parameter. Equation (15) consists two unknown constants i.e. $A_{1} \& A_{2}$ arising due to the substitution of $W$. These two constants are to be determined as follows

$\delta\left(P^{*}-\lambda^{2} K^{*}\right) / \delta A_{r_{\mathrm{t}}}, \mathrm{n}=1,2$

On simplifying (16), one gets

$C_{n_{1}} A_{1}+C_{n_{2}} A_{2}=0, \quad \mathrm{n}=1,2$

the frequency parameter.

Choosing $A_{1}=1$, one can get easily from equation (17) $A_{2}$, which is $\left(-C_{11} / C_{12}\right)$.

For a non-trivial solution, the determinant of the coefficient of equation (17) must be zero. So one gets, the frequency equation

$\left|\begin{array}{ll}C_{11} & C_{11} \\ C_{11} & C_{11}\end{array}\right|=0$ 
Indian Journal of Science and Technology

Fig. 1. Time period Vs Non homogeneity constant

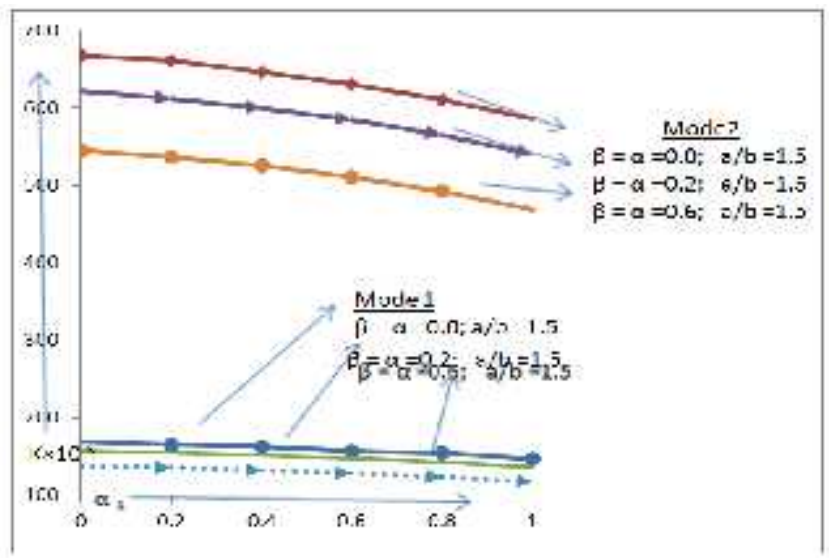

Fig. 2 Time period Vs Taper constant

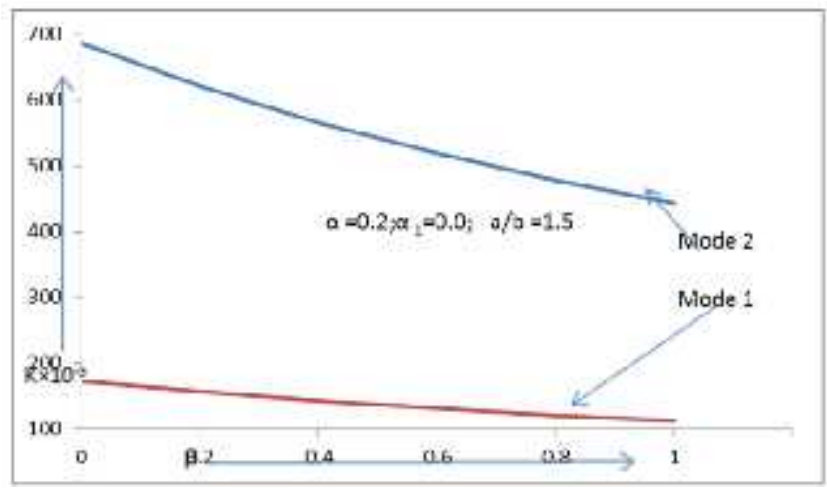

Fig. 3. Time period Vs Taper constant

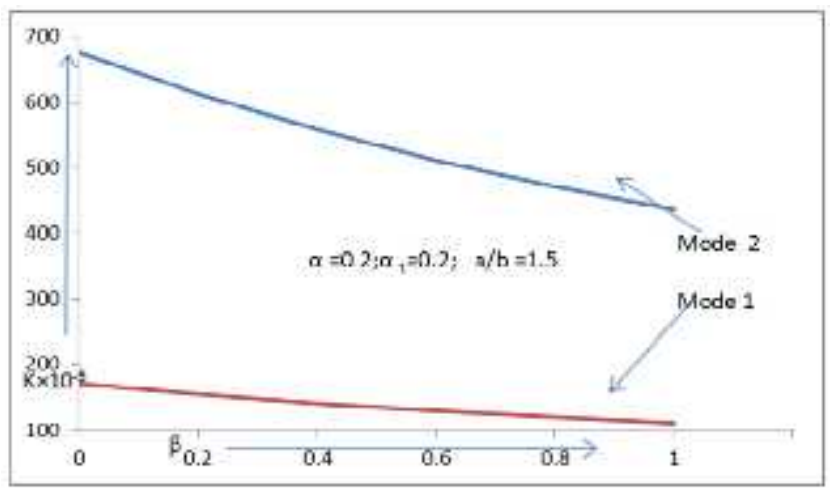

Fig. 4. Time period Vs Taper constant

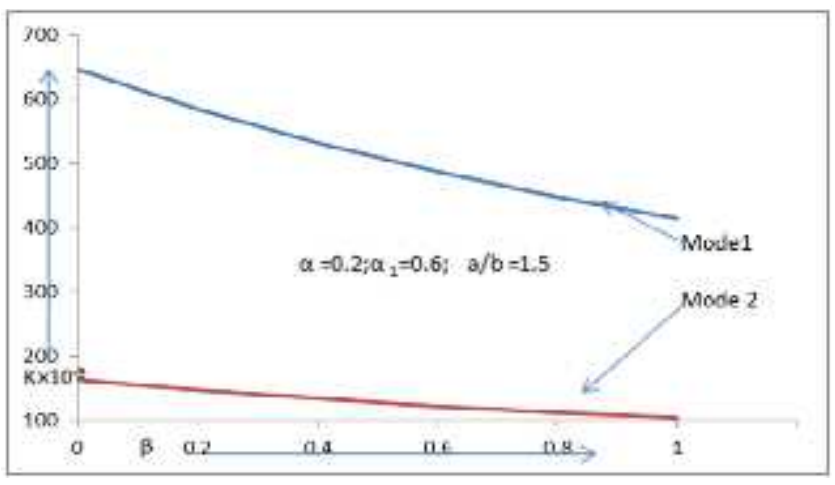

Vol. 5 No. 9 (Sep. 2012)

ISSN: 0974- 6846

Fig. 5. Time period Vs Aspect ratio

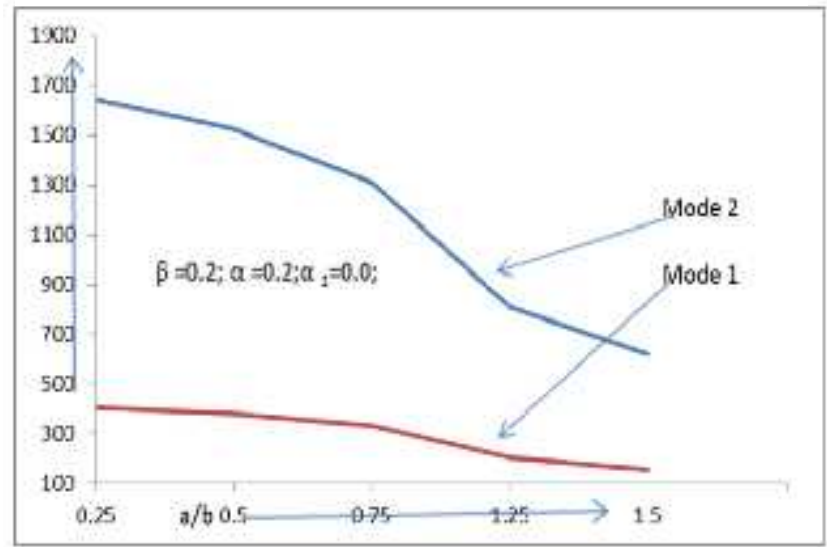

Fig. 6. Time period Vs Aspect ratio

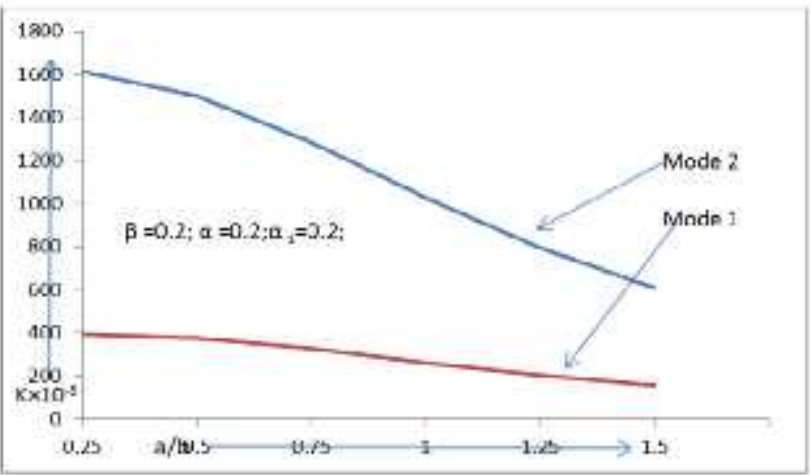

Fig. 7. Time period Vs Aspect ratio

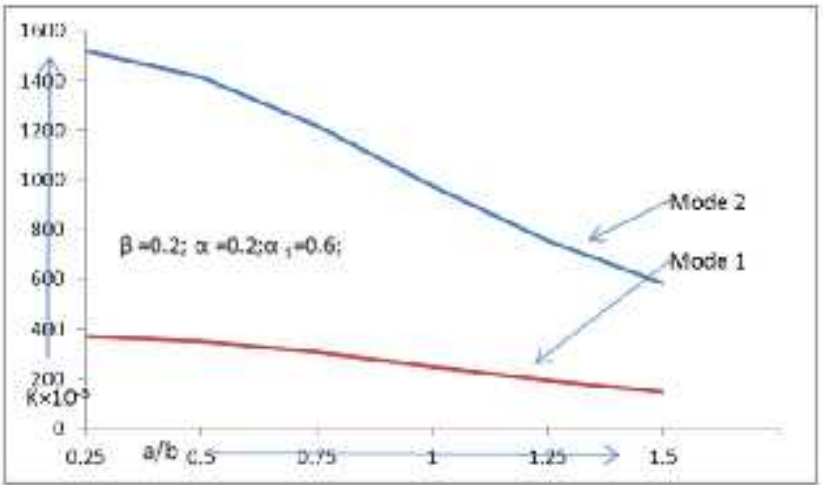

Fig. 8. Time period Vs Thermal gradient

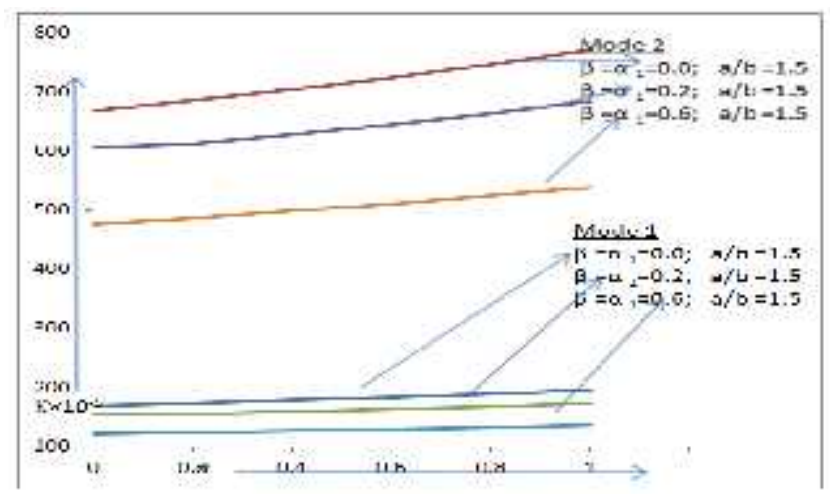

Research article CCIndian Society for Education and Environment (iSee)
A.Khanna et al. Indian J.Sci.Technol. 
From equation (17), one can obtain a quadratic equation in $\lambda^{2}$ from which the two values of $\lambda^{2}$ can found. Now with the help of the values of $A_{1}$ and $A_{2}$, one can obtain deflection function $\mathrm{W}$ as

$W=[X Y(a / b)(1-X)(1-Y a / b)]^{2}\left[1+\left(-C_{11} / C_{12}\right) X Y(a / b)(1-X)(1-\right.$ $Y a / b)]$-----(19)

Time period of the vibration of the plate is given by

$K=2 \pi / p$

where $p$ is frequency .

Results and discussion

For calculating the values of time period $(K)$ for a rectangular plate with different values of aspect ratio $(a / b)$, thermal gradient $(\alpha)$, non-homogeneity constant $\left(\alpha_{1}\right)$ and taper constant $(\beta)$ for first two modes of vibrations, the following material parameters are used which is for DURALUMIN reported at (2007):

$E=7.08 * 10^{10} \mathrm{~N} / \mathrm{M}^{2}, \rho=2.80^{*} 10^{3} \mathrm{Kg} / \mathrm{M}^{3}, \mathrm{v}_{0}=0.345$. The thickness of the plate at the center is taken as $h_{0}=0.01 \mathrm{~m}$. In the present problem, latest software technology "MATHEMATICA" is used to get the numerical results with great accuracy and concentration. Computations have been made for calculating, time period for different values of taper constant $\beta$ and aspect ratio $a / b$ for first two modes of vibration. We had considered the various cases of time period against non-homogeneity constant, taper constant, aspect ratio \& thermal gradient which are stated as below:

\section{Time period Vs Non homogeneity constant}

From Figure1, one can clearly observe that as nonhomogeneity constant $\alpha_{1}$ increases from 0.0 to 1.0 , time period decreases continuously for increasing value of taper constant and thermal gradient for first two modes of vibration.

Time period Vs Taper constant

From Figures 2, $3 \& 4$, authors conclude that time period decreases continuously as taper constant increases from 0.0 to 1.0 for fixed value of thermal gradient $(\alpha=0.2)$ for both the modes of vibrations for the following three cases:

i) $\alpha_{1}=0.0$ (Fig.2.) ii) $\alpha_{1}=0.2$ (Fig.3.) iii) $\alpha_{1}=0.6$ (Fig.4.)

On comparing these three cases, one can easily seen that as non-homogeneity constant increase, time period also decreases for both the modes of vibrations.

Time period Vs Aspect ratio

From Figures 5, 6 \& 7 one can clearly observed that time period decreases continuously as aspect ratio increases from 0.25 to 1.5 for fixed value of thermal gradient and taper constant i.e.

$\beta=0.2 ; \alpha=0.2$ for both the first two modes of vibrations for the following three cases:
i) $\alpha_{1}=0.0$ (Fig.5.)
ii) $\alpha_{1}=0.2$ (Fig.6.)
iii) $\quad \alpha_{1}$

$=0.6$ (Fig.7.)

On comparing above these three cases, one can easily found that as non-homogeneity constant increase, time period also decreases for both the first two modes of vibrations.

\section{Time period Vs Thermal gradient}

Numerical results of time period with thermal gradient are plotted in Figure 8 for different combinations of taper constant and non-homogeneity constant i.e.
i) $\beta=\alpha_{1}=0.0$
ii) $\beta=\alpha_{1}=0.2$
iii) $\beta=\alpha_{1}=0.6$

Authors can easily conclude that time period continuously increases as thermal gradient $\alpha$ increases from 0.0 to1.0 for first two modes of vibrations. It should be noted that as combined values of $\beta \& \alpha_{1}$ increase, time period is found to be decreases for both the modes of vibrations.

\section{Conclusion}

Author's motive is to provide such kind of a mathematical design so that scientist can perceive their potential in mechanical engineering field \& increase strength, durability and efficiency of mechanical design and structuring with a practical approach .Actually this is the need of the hour to develop more but authentic mathematical model for the help of mechanical engineers/researchers/practitioners. Therefore mechanical engineers and technocrats are advised to study and get the practical importance of the present paper and to provide much better structure and machines with more safety and economy.

\section{Acknowledgment}

The authors are grateful to Sh. Tarsem Garg (Hon'ble Chancellor, Maharishi Markandeshwar University, India) and Dr. Deepak Gupta (Prof.\& Head, Department of Mathematics, Maharishi Markandeshwar University, India) for their constant encouragement and also for providing all the requirements. The authors are indebted to the referees for their valuable suggestion and constructive criticism.

\section{References}

1. Cheung YK and Zhou D (1999) The free vibrations of tapered rectangular plates using a new set of beam functions with the ray liegh-ritzmethod. J. Sound \& Vibration. 223, 703-722.

2. Dhotarad MS and Ganesan N (1978) Vibration analysis of a rectangular plate subjected to a thermal gradient. J. Sound \& Vibration. 60, 481-497.

3. Gupta AK,Johri and Tripti (2007) Thermal effect on vibration of non-homogeneous orthotropic rectangular plate having bi-directional parabolically varying thickness. Proce. Intl. Conf. World Congresson Engg. \& Comput. Sci. 2007, San-Francisco, USA. pp: 24-26; 11, 784-787.

4. Gupta Dharam V, Khanna Anupam and Gupta Arun (2009) Free Vibration of clamped visco elastic rectangular plate having bi-direction exponentially thickness variation. J. Theoretical \&Appl. Mech., Warsaw. 2, 457-471.

5. Jain RK and Soni SR (1973) Free vibrations of rectangular plates of parabolically varying thickness. Indi. J. Pure Appl. Math. 4, 267-277.

6. Kumar and Sanjay (2003) Effect of thermal gradient on some vibration problems of orthotropic visco-
Research article

CCIndian Society for Education and Environment (iSee)
"Thermal gradient"

http://www.indjst.org
A.Khanna et al. Indian J.Sci.Technol. 
elastic plates of variable thickness. Ph.D. Thesis, C.C.S. Univ., Meerut, U.P., India.

7. Khanna and Anupam (2005) Some vibration problems of visco-elastic plate of variable thickness in two directions. Ph.D. Thesis, C.C.S. Univ., Meerut, U.P. India.

8. LaIRoshan and Dhanpati (2009) Effect of non homogeneity on vibration of orthotropic rectangular plates of varying thickness resting on pasternak foundation. J. Vibration \& Acoustics, ASME. 131,1-9.

9. Laura PAA, Grossi RO and Carneiro Gl (1979) Transverse vibrations of rectangular plates with thickness varying in two directions and with edges elastically restrained against rotation. J. Sound \& Vibration. 63,499-505.

10. Leissa AW (1987) Recent studies in plate vibration 1981-1985 part II, complicating effects. The Shock \& Vibration Digest. 19, 10-24.

11. Leissa AW (1987) Recent studies in plate vibration 1981-1985. Part II, complicating effects. The Shock \& Vibration Digest.19, 10-24.

12. Singh B and Saxena V (1996) Transverse vibration of rectangular plate with bidirectional thickness variation. J. Sound \& Vibration. 198, 51-65.

13. Tomar JS and Gupta AK (1983) Thermal effect on frequencies of an orthotrophic rectangular plate of linearly varying thickness. J. Sound \& Vibration. 90(3), 325-331.

14. Tomar S and Gupta AK (1985) Effect of thermal gradient on frequencies of an orthotropic rectangular plate whose thickness varies in two directions. J. Sound \& Vibration. 98, 257-262.

15. Li WL (2004) Vibration analysis of rectangular plate with general elastic boundary supports. J. Sound \& Vibration. 273(3), 619-635. 